\title{
Incompressible Viscous Flows in Borderline Besov Spaces
}

\author{
TAOufiK Hmidi \& Sahbi KeraAni
}

\begin{abstract}
We establish two new estimates for a transport-diffusion equation. As an application we treat the problem of global persistence of the Besov regularity $B_{p, 1}^{\frac{2}{p}+1}$, with $p \in] 2,+\infty$ ], for the two-dimensional Navier-Stokes equations with uniform bounds on the viscosity. We provide also an inviscid global result.
\end{abstract}

\section{Introduction}

In this paper we are concerned with the incompressible Navier-Stokes equations governing a viscous fluid evolving in the whole space $\mathbb{R}^{d}$,

$$
\left(N S_{\mu}\right) \quad\left\{\begin{array}{l}
\partial_{t} v_{\mu}+v_{\mu} \cdot \nabla v_{\mu}-\mu \Delta v_{\mu}=-\nabla \pi_{\mu} \\
\operatorname{div} v_{\mu}=0 \\
v_{\mu}(0, x)=v^{0}(x)
\end{array}\right.
$$

Here, the vector field $v_{\mu}(t, x)=\left(v_{\mu}^{1}, \ldots, v_{\mu}^{d}\right)(t, x)$ stands for the velocity of the fluid, the scalar $\pi_{\mu}$ denotes the pressure and the parameter $\mu>0$ is the kinematic viscosity. We will also consider the Euler equations which are the inviscid case of the system $\left(N S_{\mu}\right)$,

$$
(E) \quad\left\{\begin{array}{l}
\partial_{t} v+v \cdot \nabla v=-\nabla \pi \\
\operatorname{div} v=0 \\
v(0, x)=v^{0}(x)
\end{array}\right.
$$

The Cauchy problem for Navier-Stokes system has been intensively investigated since the pioneering work of LERAY [12] who proved the existence of global weak solutions in the energy space. Nevertheless, the uniqueness of such solutions is only known in space dimension two and is still a widely open problem for higher dimension ( $d \geqq 3$ ). For strong solutions, Fusita and KATo [7] proved a local 
well-posedness result when the initial data are lying in the homogeneous Sobolev space $\dot{H}^{\frac{d}{2}-1}$, which is invariant under the scaling of the equations. It is worth pointing out that the same result holds true when the initial data belong to the inhomogeneous Sobolev space $H^{s}$, with $s \geqq \frac{d}{2}-1$. However the problem of whether these solutions blow up in finite time or not is still unsolved and is considered as one of the most relevant problem of the nonlinear PDE's. We emphasize that the global existence is only known in some restrictive cases as for example in space dimension two or when the initial data are small in some function spaces which are invariant under the scaling.

For a Euler system the theory is widely developed, and we restrict our attention to some significant results. In [11] KATO and PONCE proved a local well-posedness result in $H^{s}$ with $s>\frac{d}{2}+1$. In space dimension two such solutions are global since the vorticity does not concentrate in finite time, see for instance [2]. It seems very hard to obtain a local well-posedness result when initial data belong to the critical space $H^{1+\frac{d}{2}}$ because we lack the embedding in Lipschitz functions. Nevertheless local results are given when we work with Besov spaces $B_{p, 1}^{1+\frac{d}{p}}$, with $\left.p \in\right] 1, \infty[$, see [3]. Recently, VISHIK has proved in [15] that these solutions are global in dimension two. His proof relies on a subtle logarithmic estimate based on the explicit formula of the vorticity in dimension two. We mention that we have extended in [10] the global existence in the limiting case $p=+\infty$.

The inviscid limit was performed by several authors and is well understood. For example, it is proven in [13] that under the assumption $v^{0} \in H^{s}$ with $s>\frac{d}{2}+2$, the solutions $\left(v_{\mu}\right)_{\mu>0}$ converge in $L^{2}$ norm as $\mu \rightarrow 0$ to the unique solution $v$ of $(E)$ and the convergence rate is of order $\mu t$. We point out that in dimension two these results are global in time.

This paper is the sequel to [9] in which we continue to study essentially two problems. The first one is the uniform persistence with respect to vanishing viscosity of viscous solutions in critical Besov spaces $B_{p, 1}^{2 / p+1}\left(\mathbb{R}^{2}\right)$, whereas the second one deals with the inviscid limit and the rate convergence. In [9] we have given an affirmative answer when $p \in] 1,2]$ and the crucial fact of the proof is a new regularization effect of the vorticity equation:

$$
\partial_{t} \omega_{\mu}+v_{\mu} \cdot \nabla \omega_{\mu}-\mu \Delta \omega_{\mu}=0 \text { with } \omega_{\mu}=\partial_{1} v_{\mu}^{2}-\partial_{2} v_{\mu}^{1} .
$$

We recall the following identity:

$$
v_{\mu}=\Delta^{-1} \nabla^{\perp} \omega_{\mu} \quad \text { with } \nabla^{\perp}=\left(-\partial_{2}, \partial_{1}\right) .
$$

We have established in [9] the following linear estimate on the Lipschitz norm of the velocity

$$
\mu\left\|\int_{0}^{t} \omega_{\mu}(\tau) \mathrm{d} \tau\right\|_{B_{\infty, \infty}^{2}} \leqq C\left\|\omega^{0}\right\|_{L^{\infty}}\left(1+\int_{0}^{t}\left\|\nabla v_{\mu}(\tau)\right\|_{L^{\infty}} \mathrm{d} \tau\right),
$$

which is essential to bound uniformly on $\mu$ the quantity $\left\|\nabla v_{\mu}\right\|_{L_{+}^{1} L^{\infty}}$ (for the definition of Besov spaces we refer the reader to the next section). This method fails for $p>2$, and we are led to use an other approach based on a new logarithmic estimate. Our main result reads as follows. 
Theorem 1. Let $p \in] 2, \infty]$ and $v^{0}$ be a divergence free vector field belonging to $B_{p, 1}^{\frac{2}{p}+1}\left(\mathbb{R}^{2}\right)$. Then the Navier-Stokes system $\left(\mathrm{NS}_{\mu}\right)$ has a unique global solution in $\mathscr{C}\left(\mathbb{R}_{+} ; B_{p, 1}^{\frac{2}{p}+1}\right)$, satisfying in addition the following uniform estimate:

$$
\left\|v_{\mu}(t)\right\|_{B_{p, 1}^{\frac{2}{p}+1}} \leqq C_{0} e^{\exp C_{0} t}
$$

Moreover, the family $\left(v_{\mu}\right)_{\mu>0}$ converges to Euler solution $v$ with the following rate:

$$
\left.\left.\left\|v_{\mu}(t)-v(t)\right\|_{L^{p}} \leqq C_{0} e^{\exp C_{0} t}(\mu t)^{\frac{1}{2}+\frac{1}{p}}, \quad \forall \mu \in\right] 0,1\right] .
$$

Here, $C_{0}$ is a constant depending only on the initial data but not on the viscosity.

Remark 1. In the above theorem the existence and uniqueness of global solutions is well-known, and the novelty of this result is the uniform estimates on the viscosity and the inviscid limit result.

The proof of Theorem 1 relies on some new estimates for the transport-diffusion equation:

$$
\left(\mathrm{TD}_{\mu}\right) \quad\left\{\begin{array}{l}
\partial_{t} a+v \cdot \nabla a-\mu \Delta a=f \\
a(0, x)=a^{0}(x)
\end{array}\right.
$$

The first estimate is a new logarithmic estimate for the solution of $\left(\mathrm{TD}_{\mu}\right)$, which is an extension of VISHIK's one [15] obtained for the inviscid case $(\mu=0)$.

Theorem 2. Let $p \in[1,+\infty], v$ be a divergence-free vector field belonging to the space $L_{\mathrm{loc}}^{1}\left(\mathbb{R}_{+} ; \operatorname{Lip}\left(\mathbb{R}^{d}\right)\right)$ and $f \in L_{\text {loc }}^{1}\left(\mathbb{R}_{+} ; B_{p, 1}^{0}\right)$. Then any smooth scalar solution a to the problem $\left(\mathrm{TD}_{\mu}\right)$ satisfies for all $t \in \mathbb{R}_{+}$

$$
\|a(t)\|_{B_{p, 1}^{0}} \leqq C\left(\left\|a^{0}\right\|_{B_{p, 1}^{0}}+\|f\|_{L_{t}^{1} B_{p, 1}^{0}}\right)\left(1+\int_{0}^{t}\|\nabla v(\tau)\|_{L^{\infty}} d \tau\right),
$$

where $C$ is a constant depending only on d but not on the viscosity.

Remark 2. Let us note that the logarithmic estimate allows us to bound uniformly with respect to $\mu$ the Lipschitz norm of the Navier-Stokes velocity, and this fact is crucial for the global persistence of the initial regularity.

Remark 3. Vishik's proof is widely dependent on the structure of the equation. Indeed it uses the fact that the solution for transport equation is explicitly expressed through the initial data and the flow, and clearly this fails for the system $\left(\mathrm{TD}_{\mu}\right)$. However, as in the proof of VISHIK [15], we take account in a crucial way of the incompressibility of the flow.

Our second estimate is some smoothing effect for $\left(\mathrm{TD}_{\mu}\right)$. It will be useful to establish a better rate of convergence for the inviscid limit. 
Theorem 3. Let $s \in]-1,1\left[,\left(p_{1}, p_{2}, r\right) \in[1,+\infty]^{3}\right.$ and $v$ be a divergence free vector field belonging to $L_{\text {loc }}^{1}\left(\mathbb{R}_{+} ; \operatorname{Lip}\left(\mathbb{R}^{d}\right)\right)$. Then, there exists a constant $C=$ $C(d, s)$ such that the following holds true: let a to be a smooth solution for $\left(\mathrm{TD}_{\mu}\right)$, then

$\mu^{\frac{1}{r}}\|a\|_{\widetilde{L_{t}^{r}} B_{p_{1}, p_{2}}^{s+\frac{2}{r}}} \leqq C e^{C V(t)}(1+\mu t)^{\frac{1}{r}}\left(\left\|a^{0}\right\|_{B_{p_{1}, p_{2}}^{s}}+\|f\|_{L_{t}^{1} B_{p_{1}, p_{2}}^{s}}\right), \quad \forall t \in \mathbb{R}_{+}$,

with $V(t)=\int_{0}^{t}\|\nabla v(\tau)\|_{L^{\infty}} d \tau$.

Here our estimates are described through a coupled space $\widetilde{L_{t}^{r}} B_{p_{1}, p_{2}}^{s}$ which is slightly different from the usual space $L_{t}^{r} B_{p_{1}, p_{2}}^{s}$. For more precise details, we refer the reader to the next section.

Remark 4. In the above theorem both cases $r=+\infty$ and $p_{1}<+\infty$ are wellknown. The first one can be proven by standard method but the second is more subtle and was established by DANCHIN [6] with a blowing-up constant $C$ as $p_{1}$ goes to $+\infty$. Thus the critical situation $p_{1}=\infty$ seems to be not reached by these methods. In [8] the first author gave a positive answer for the Hölderian spaces $\left(p_{1}=p_{2}=\infty\right)$ by using another approach based on Lagrangian coordinates. Here we improve this result by obtaining a better control on the right-hand side of the estimate of Theorem 3 .

The rest of this paper is organized as follows. We collect in Section 2 some basic results of Littlewood-Paley theory, and we provide some useful lemmas. In Section 3, we prove the logarithmic estimate and the Section 4 is reserved to the proof of Theorem 3. We discuss in Section 5 our Theorem 1 and finally we conclude this paper by an Appendix.

\section{Notation and preliminaries}

Throughout this paper we use the notation $X \precsim Y$ to denote the estimate $X \leqq C Y$ for some constant $0<C<\infty$ which depends only on the dimension. The notation $C_{0}$ stands for a real positive constant depending on the initial data.

To define Besov spaces we shall need the dyadic decomposition. We recall that there exists two radially functions $\chi \in \mathscr{D}\left(\mathbb{R}^{d}\right)$ and $\varphi \in \mathscr{D}\left(\mathbb{R}^{d} \backslash\{0\}\right)$ such that

$$
\chi(\xi)+\sum_{q \geqq 0} \varphi\left(2^{-q} \xi\right)=1 .
$$

For every tempered distribution $v \in \mathscr{S}^{\prime}$ we set

$$
\Delta_{-1} v=\chi(\mathrm{D}) v ; \forall q \in \mathbb{N}, \quad \Delta_{q} v=\varphi\left(2^{-q} \mathrm{D}\right) v \quad \text { and } \quad S_{q}=\sum_{j=-1}^{q-1} \Delta_{j} .
$$

We will need also the homogeneous operators:

$$
\dot{\Delta}_{q} v=\varphi\left(2^{-q} \mathrm{D}\right) v, \quad \forall q \in \mathbb{Z} .
$$


The paradifferential calculus introduced by BoNY [1] is based on the following decomposition (called Bony's decomposition) which split the product $u v$ into three parts:

$$
u v=T_{u} v+T_{v} u+R(u, v)
$$

with

$$
T_{u} v=\sum_{q} S_{q-1} u \Delta_{q} v \text { and } R(u, v)=\sum_{\left|q^{\prime}-q\right| \leqq 1} \Delta_{q} u \Delta_{q^{\prime}} v
$$

Let us now recall the definition of Besov spaces through dyadic decomposition. Let $\left(p_{1}, p_{2}\right) \in[1,+\infty]^{2}$ and $s \in \mathbb{R}$, then the space $B_{p_{1}, p_{2}}^{s}$ is the set of tempered distribution $u$ such that

$$
\|u\|_{B_{p_{1}, p_{2}}^{s}}:=\left(2^{q s}\left\|\Delta_{q} u\right\|_{L^{p_{1}}}\right)_{\ell^{p_{2}}}<+\infty .
$$

We define also the homogeneous norm:

$$
\|u\|_{\dot{B}_{p_{1}, p_{2}}^{s}}:=\left(2^{q s}\left\|\dot{\Delta}_{q} u\right\|_{L^{p_{1}}}\right)_{\ell^{p_{2}}} .
$$

The definition of Besov spaces does not depend on the choice of the dyadic decomposition. One can further remark that Sobolev space $H^{s}$ coincide with $B_{2,2}^{s}$.

Our study will require the use of the following coupled spaces. Let $T>0$ and $r \geqq 1$, we denote by $L_{T}^{r} B_{p_{1}, p_{2}}^{s}$ the space of all function $u$ satisfying

$$
\|u\|_{L_{T}^{r} B_{p_{1}, p_{2}}^{s}}:=\left\|\left(2^{q s}\left\|\Delta_{q} u\right\|_{L^{p_{1}}}\right)_{\ell p_{2}}\right\|_{L_{T}^{r}}<\infty
$$

We say that a function $u$ is an element of the space $\widetilde{L_{T}^{r}} B_{p_{1}, p_{2}}^{s}$ if

$$
\|u\|_{\widetilde{L_{T}^{r}} B_{p_{1}, p_{2}}^{s}}:=\left(2^{q s}\left\|\Delta_{q} u\right\|_{L_{T}^{r} L^{p_{1}}}\right)_{\ell^{p_{2}}}<+\infty .
$$

The relationships between these spaces are described as follows and they are a direct consequence of Minkowski inequalities:

$$
\begin{array}{ll}
L_{T}^{r} B_{p_{1}, p_{2}}^{s} \hookrightarrow \widetilde{L_{T}^{r}} B_{p_{1}, p_{2}}^{s}, & \text { if } r \leqq p_{2} ; \\
\widetilde{L_{T}^{r}} B_{p_{1}, p_{2}}^{s} \hookrightarrow L_{T}^{r} B_{p_{1}, p_{2}}^{s}, & \text { if } r \geqq p_{2} .
\end{array}
$$

We have also the following real interpolation result that will be used in the proof of the inviscid limit. Let $T>0$ and $s_{1}<s<s_{2}$ and $\left.\theta \in\right] 0,1[$ such that $s=\theta s_{1}+(1-\theta) s_{2}$. Then we have

$$
\|u\|_{\widetilde{L_{T}^{r}}} B_{p_{1}, p_{2}}^{s} \leqq C\|u\|_{\widetilde{L_{T}^{r}}}^{\theta} B_{p_{1}, \infty}^{s_{1}}\|u\| \underset{\widetilde{L_{T}^{r}} B_{p_{1}, \infty}^{s_{2}}}{1-},
$$

for some absolute constant $C$.

In our proofs we will make an intensive use of the following well-known inequalities. 
Lemma 1. (Bernstein) There exists $C>0$ such that $\forall q \in \mathbb{Z}$, and for $a \geqq b \geqq 1$, we have

$$
C^{-k} 2^{q k}\left\|\dot{\Delta}_{q} u\right\|_{L^{a}} \leqq \sup _{|\alpha|=k}\left\|\partial^{\alpha} \dot{\Delta}_{q} u\right\|_{L^{a}} \leqq C^{k} 2^{q\left(k+d\left(\frac{1}{b}-\frac{1}{a}\right)\right)}\left\|\dot{\Delta}_{q} u\right\|_{L^{b}}
$$

Now, we recall the following commutator lemma (see [4] and the references therein).

Lemma 2. Let $v$ be a Lipschitz divergence free vector field of $\mathbb{R}^{d}$ and $\left(s, p_{1}, p_{2}\right) \in$ ]$-1,1\left[\times[1,+\infty]^{2}\right.$. Then, there exists a constant $C$ depending only on $s$ and $d$, such that

$$
\left\|2^{q s}\right\|\left[\Delta_{q}, v \cdot \nabla\right] a\left\|_{L^{p_{1}}}\right\|_{\ell^{p_{2}}} \leqq C\|\nabla v\|_{L^{\infty}\|a\|_{B_{p_{1}, p_{2}}^{s}}}
$$

To finish this paragraph we state the following result which can be found in $[4,6]$.

Proposition 1. Let $s \in(-1,1),\left(p_{1}, p_{2}, r\right) \in[1, \infty]^{3}$ and $v$ be a divergence free vector field belonging to $L_{\text {loc }}^{1}\left(\mathbb{R}_{+} ; \operatorname{Lip}\left(\mathbb{R}^{d}\right)\right)$. Let a be a solution of

$$
\partial_{t} a+v \cdot \nabla a-\mu \Delta a=f .
$$

Then the following estimate holds true

$$
\|a(t)\|_{B_{p_{1}, p_{2}}^{s}} \leqq C e^{C V(t)}\left(\|a(0)\|_{B_{p_{1}, p_{2}}^{s}}+\int_{0}^{t} e^{-C V(\tau)}\|f(\tau)\|_{B_{p_{1}, p_{2}}^{s}} d \tau\right),
$$

with $V(t)=\int_{0}^{t}\|\nabla v(\tau)\|_{L^{\infty}} d \tau$ and $C$ a constant depending only on $s$ and $d$.

\section{Proof of Theorem 2}

For every $q \in \mathbb{N} \cup\{-1\}$ we will denote by $\tilde{a}_{q}$ the unique global solution of the initial value problem

$$
\left\{\begin{array}{l}
\partial_{t} \tilde{a}_{q}+v \cdot \nabla \tilde{a}_{q}-\mu \Delta \tilde{a}_{q}=f_{q}:=\Delta_{q} f \\
\tilde{a}_{q}(0, \cdot)=\Delta_{q} a^{0}
\end{array}\right.
$$

According to Proposition 1, we have

$$
\left\|\tilde{a}_{q}(t)\right\|_{B_{p, \infty}^{ \pm \varepsilon}} \leqq C_{\varepsilon}\left(\left\|\Delta_{q} a^{0}\right\|_{B_{p, \infty}^{ \pm \varepsilon}}+\left\|f_{q}\right\|_{L_{t}^{1} B_{p, \infty}^{ \pm \varepsilon}}\right) e^{C V(t)}, \quad \forall 0 \leqq \varepsilon<1 .
$$

Here and throughout this paper we use the notation

$$
V(t):=\|\nabla v\|_{L_{t}^{1} L^{\infty}}
$$

Combined with the definition of Besov spaces this yields for $j, q \geqq-1$,

$$
\left\|\Delta_{j} \tilde{a}_{q}(t)\right\|_{L^{p}} \leqq C_{\varepsilon} 2^{-\varepsilon|j-q|}\left(\left\|\Delta_{q} a^{0}\right\|_{L^{p}}+\left\|f_{q}\right\|_{L_{t}^{1} L^{p}}\right) e^{C V(t)} .
$$


By linearity it is obvious that $a=\sum_{q=-1}^{\infty} \tilde{a}_{q}$. Thus we get from the definition of Besov spaces

$$
\|a(t)\|_{B_{p, 1}^{0}} \leqq \sum_{|j-q| \geqq N}\left\|\Delta_{j} \tilde{a}_{q}(t)\right\|_{L^{p}}+\sum_{|j-q|<N}\left\|\Delta_{j} \tilde{a}_{q}(t)\right\|_{L^{p}},
$$

where $N \in \mathbb{N}$ is to be chosen later. To deal with the first sum we use (5) with $\varepsilon=1 / 2$

$$
\begin{aligned}
\sum_{|j-q| \geqq N}\left\|\Delta_{j} \tilde{a}_{q}(t)\right\|_{L^{p}} & \precsim 2^{-N / 2} \sum_{q}\left(\left\|\Delta_{q} a^{0}\right\|_{L^{p}}+\left\|f_{q}\right\|_{L_{t}^{1} L^{p}}\right) e^{C V(t)} \\
& \precsim 2^{-N / 2}\left(\left\|a^{0}\right\|_{B_{p, 1}^{0}}+\|f\|_{L_{t}^{1} B_{p, 1}^{0}}\right) e^{C V(t)} .
\end{aligned}
$$

We now turn to the second sum in the right-hand side of (6).

On the one hand, since the operator $\Delta_{j}$ maps $L^{p}$ into itself uniformly with respect to $j$, one has

$$
\sum_{|j-q|<N}\left\|\Delta_{j} \tilde{a}_{q}(t)\right\|_{L^{p}} \precsim \sum_{|j-q|<N}\left\|\tilde{a}_{q}(t)\right\|_{L^{p}} .
$$

On the other hand, a standard $L^{p}$ energy estimate of the system (4) yields

$$
\left\|\tilde{a}_{q}(t)\right\|_{L^{p}} \leqq\left\|a_{q}(0)\right\|_{L^{p}}+\left\|f_{q}\right\|_{L_{t}^{1} L^{p}} .
$$

So, it holds that

$$
\sum_{|j-q|<N}\left\|\Delta_{j} \tilde{a}_{q}(t)\right\|_{L^{p}} \precsim N\left(\left\|a^{0}\right\|_{B_{p, 1}^{0}}+\|f\|_{L_{t}^{1} B_{p, 1}^{0}}\right) .
$$

The outcome is the following

$$
\|a(t)\|_{B_{p, 1}^{0}} \precsim\left(\left\|a^{0}\right\|_{B_{p, 1}^{0}}+\|f\|_{L_{t}^{1} B_{p, 1}^{0}}\right)\left(2^{-N / 2} e^{C V(t)}+N\right) .
$$

Choosing

$$
N=\left[\frac{2 C V(t)}{\log 2}+1\right]
$$

we get the desired result.

Remark 5. We deduce from (5) $(\mu=0)$ that for any $a^{0} \in L^{p}$ and for any flow $\psi$ corresponding to a time-dependent smooth velocity,

$$
\forall j, q \geqq-1, \quad\left\|\Delta_{j}\left(\Delta_{q}\left(a^{0}\right) \circ \psi(t)\right)\right\|_{L^{p}} \leqq C 2^{-\varepsilon|j-q|}\left\|\Delta_{q} a^{0}\right\|_{L^{p}} e^{C V(t)},
$$

for every $\varepsilon \in[0,1$ [ and $C$ depends on $\varepsilon$. This estimate was proved by VISHIK [15] with $\varepsilon=1$ :

$$
\left\|\Delta_{j}\left(\Delta_{q}\left(a^{0}\right) \circ \psi(t)\right)\right\|_{L^{p}} \leqq C 2^{-|j-q|}\left\|\Delta_{q} a^{0}\right\|_{L^{p}} e^{C V(t)} .
$$




\section{Proof of Theorem 3}

We give the proof only for $r=+\infty$ and $r=1$. The general case is obtained by an easy argument of interpolation.

The Fourier localized function $a_{q}:=\Delta_{q} a$ satisfies

$$
\partial_{t} a_{q}+v \cdot \nabla a_{q}-\mu \Delta a_{q}=-\left[\Delta_{q}, v \cdot \nabla\right] a+f_{q} .
$$

A standard $L^{p_{1}}$ estimate gives

$$
\left\|a_{q}(t)\right\|_{L_{t}^{\infty} L^{p_{1}}} \leqq\left\|a_{q}(0)\right\|_{L^{p_{1}}}+\int_{0}^{t}\left\|\left[\Delta_{q}, v \cdot \nabla\right] a(\tau)\right\|_{L^{p_{1}}} \mathrm{~d} \tau+\int_{0}^{t}\left\|f_{q}(\tau)\right\|_{L^{p_{1}}} \mathrm{~d} \tau .
$$

Multiplying by $2^{q s}$ and taking the $\ell^{p_{2}}$ norm, we obtain, via Minkowski inequality and Lemma 2,

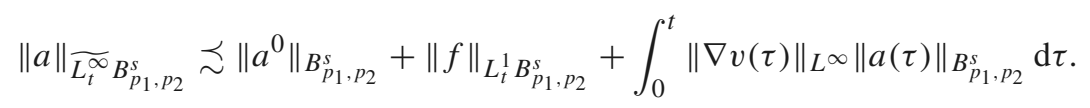

The required estimate follows from Gronwall's lemma.

We now turn to the case $r=1$. We start by rewriting (9) as

$\partial_{t} a_{q}+S_{q-1} v \cdot \nabla a_{q}-\mu \Delta a_{q}=\left(S_{q-1}-\mathrm{Id}\right) v \cdot \nabla a_{q}-\left[\Delta_{q}, v \cdot \nabla\right] a+f_{q}:=g_{q}$.

Let $\psi_{q}$ denote the flow of the regularized velocity $S_{q-1} v$ :

$$
\psi_{q}(t, x)=x+\int_{0}^{t} S_{q-1} v\left(\tau, \psi_{q}(\tau, x)\right) \mathrm{d} \tau .
$$

We set

$$
\bar{a}_{q}(t, x)=a_{q}\left(t, \psi_{q}(t, x)\right) \text { and } \bar{g}_{q}(t, x)=g_{q}\left(t, \psi_{q}(t, x)\right)
$$

From Leibnitz formula we deduce the following identity

$$
\Delta \bar{a}_{q}(t, x)=\sum_{i=1}^{d}\left\langle H_{q} \cdot\left(\partial^{i} \psi_{q}\right)(t, x),\left(\partial^{i} \psi_{q}\right)(t, x)\right\rangle+\left(\nabla a_{q}\right)\left(t, \psi_{q}(t, x)\right) \cdot \Delta \psi_{q}(t, x),
$$

where $H_{q}(t, x):=\left(\nabla^{2} a_{q}\right)\left(t, \psi_{q}(t, x)\right)$ is the Hessian matrix.

Straightforward computations based on the definition of the flow and Gronwall's inequality yield

$$
\partial^{i} \psi_{q}(t, x)=e_{i}+h_{q}^{i}(t, x)
$$

where $\left(e_{i}\right)_{i=1}^{d}$ is the canonical basis of $\mathbb{R}^{d}$ and the function $h_{q}^{i}$ is estimated as follows

$$
\left\|h_{q}^{i}(t)\right\|_{L^{\infty}} \precsim V(t) e^{C V(t)}, \quad \text { with } \quad V(t):=\int_{0}^{t}\|\nabla v(\tau)\|_{L^{\infty}} \mathrm{d} \tau .
$$


Applying Leibnitz formula and Bernstein inequality, we find

$$
\left\|\Delta \psi_{q}(t)\right\|_{L^{\infty}} \precsim 2^{q} V(t) e^{C V(t)} .
$$

The outcome is

$$
\Delta \bar{a}_{q}(t, x)=\left(\Delta a_{q}\right)\left(t, \psi_{q}(t, x)\right)-\mathscr{R}_{q}(t, x),
$$

with

$$
\begin{aligned}
\left\|\mathscr{R}_{q}(t)\right\|_{L^{p_{1}}} \precsim & \left\|\nabla a_{q}(t)\right\|_{L^{p_{1}}}\left\|\Delta \psi_{q}(t)\right\|_{L^{\infty}} \\
& +\left\|\nabla^{2} a_{q}(t)\right\|_{L^{p_{1}}} \sup _{i}\left(\left\|h_{q}^{i}(t)\right\|_{L^{\infty}}+\left\|h_{q}^{i}(t)\right\|_{L^{\infty}}^{2}\right) \\
\precsim & 2^{2 q} V(t) e^{C V(t)}\left\|a_{q}(t)\right\|_{L^{p_{1}}} .
\end{aligned}
$$

In the last line we have used Bernstein inequality and the fact that the flow preserves Lebesgue measure.

From (10) and (13) we see that $\bar{a}_{q}$ satisfies

$$
\left(\partial_{t}-\mu \Delta\right) \bar{a}_{q}(t, x)=\mu \mathscr{R}_{q}(t, x)+\bar{g}_{q}(t, x) .
$$

Now, we will again localize in frequency this equation through the operator $\Delta_{j}$. So we write from Duhamel formula,

$$
\begin{aligned}
\Delta_{j} \bar{a}_{q}(t, x)= & e^{\mu t \Delta} \Delta_{j} a_{q}(0)+\mu \int_{0}^{t} e^{\mu(t-\tau) \Delta} \Delta_{j} \mathscr{R}_{q}(\tau, x) \mathrm{d} \tau \\
& +\int_{0}^{t} e^{\mu(t-\tau) \Delta} \Delta_{j} \bar{g}_{q}(\tau, x) \mathrm{d} \tau .
\end{aligned}
$$

At this stage we need the following lemma (see for instance [5]).

Lemma 3. For $u \in L^{p_{1}}$ and $j \in \mathbb{N}$,

$$
\left\|e^{\mu t \Delta} \Delta_{j} u\right\|_{L^{p_{1}}} \leqq C e^{-c \mu t 2^{2 j}}\left\|\Delta_{j} u\right\|_{L^{p_{1}}},
$$

where the constants $C$ and $c$ depend only on the dimension $d$.

Combined with (14) this lemma yields, for every $j \in \mathbb{N}$,

$$
\left\|e^{\mu(t-\tau) \Delta} \Delta_{j} \mathscr{R}_{q}(\tau)\right\|_{L^{p_{1}}} \precsim 2^{2 q} V(\tau) e^{C V(\tau)} e^{-c \mu(t-\tau) 2^{2 j}}\left\|a_{q}(\tau)\right\|_{L^{p_{1}}} .
$$

Also with the incompressibility of the flow it gives

$$
\left\|e^{\mu(t-\tau) \Delta} \Delta_{j} \bar{g}_{q}(\tau)\right\|_{L^{p_{1}}} \precsim e^{-c \mu(t-\tau) 2^{2 j}}\left(\left\|\left[\Delta_{q}, v \cdot \nabla\right] a(\tau)\right\|_{L^{p_{1}}}+\left\|f_{q}(\tau)\right\|_{L^{p_{1}}}\right) .
$$

Thus, we get from (15)

$$
\begin{aligned}
\left\|\Delta_{j} \bar{a}_{q}(t)\right\|_{L^{p_{1}}} \precsim & e^{-c \mu t 2^{2 j}}\left\|\Delta_{j} a_{q}^{0}\right\|_{L^{p_{1}}}+\int_{0}^{t} e^{-c \mu(t-\tau) 2^{2 j}}\left\|f_{q}(\tau)\right\|_{L^{p_{1}}} \mathrm{~d} \tau \\
& +V(t) e^{C V(t)} \mu 2^{2 q} \int_{0}^{t} e^{-c \mu(t-\tau) 2^{2 j}}\left\|a_{q}(\tau)\right\|_{L^{p_{1}}} \mathrm{~d} \tau \\
& +\int_{0}^{t} e^{-c \mu(t-\tau) 2^{2 j}}\left\|\left[\Delta_{q}, v \cdot \nabla\right] a(\tau)\right\|_{L^{p_{1}}} \mathrm{~d} \tau
\end{aligned}
$$


Integrating in time and using Young inequalities, we obtain for all $j \in \mathbb{N}$

$$
\begin{aligned}
\left\|\Delta_{j} \bar{a}_{q}\right\|_{L_{t}^{1} L^{p_{1}}} \precsim & \left(\mu 2^{2 j}\right)^{-1}\left(\left\|\Delta_{j} a_{q}^{0}\right\|_{L^{p_{1}}}+\left\|f_{q}\right\|_{L_{t}^{1} L^{p_{1}}}+\left\|\left[\Delta_{q}, v . \nabla\right] a\right\|_{L_{t}^{1} L^{p_{1}}}\right) \\
& +V(t) e^{C V(t)} 2^{2(q-j)} \mu\left\|a_{q}\right\|_{L_{t}^{1} L^{p_{1}}} .
\end{aligned}
$$

Let $N$ be an integer that will be fixed later. Since the flow preserves Lebesgue measure, then we have

$$
\begin{aligned}
\mu 2^{q(s+2)}\left\|a_{q}\right\|_{L_{t}^{1} L^{p_{1}}} & =\mu 2^{q(s+2)}\left\|\bar{a}_{q}\right\|_{L_{t}^{1} L^{p_{1}}} \\
& \leqq \mu 2^{q(s+2)}\left(\sum_{|j-q|<N}\left\|\Delta_{j} \bar{a}_{q}\right\|_{L_{t}^{1} L^{p_{1}}}+\sum_{|j-q| \geqq N}\left\|\Delta_{j} \bar{a}_{q}\right\|_{L_{t}^{1} L^{p_{1}}}\right) .
\end{aligned}
$$

Hence for all $q>N$, one has

$$
\begin{aligned}
\mu 2^{q(s+2)}\left\|a_{q}\right\|_{L_{t}^{1} L^{p_{1}}} \precsim & 2^{q s}\left\|a_{q}^{0}\right\|_{L^{p_{1}}}+2^{N} 2^{q s}\left\|f_{q}\right\|_{L_{t}^{1} L^{p_{1}}} \\
& +V(t) e^{C V(t)} 2^{2 N} \mu 2^{q(s+2)}\left\|a_{q}\right\|_{L_{t}^{1} L^{p_{1}}} \\
& +2^{2 N} \mu 2^{q s}\left\|\left[\Delta_{q}, v \cdot \nabla\right] a\right\|_{L_{t}^{1} L^{p_{1}}} \\
& +\mu 2^{q(s+2)} \sum_{|j-q| \geqq N}\left\|\Delta_{j} \bar{a}_{q}\right\|_{L_{t}^{1} L^{p_{1}}} .
\end{aligned}
$$

According to (8) we have

$$
\left\|\Delta_{j} \bar{a}_{q}(t)\right\|_{L^{p}} \precsim 2^{-|j-q|} e^{C V(t)}\left\|a_{q}(t)\right\|_{L^{p}} .
$$

Thus, we infer

$$
\begin{aligned}
\mu 2^{q(s+2)}\left\|a_{q}\right\|_{L_{t}^{1} L^{p_{1}}} \precsim & 2^{q s}\left\|a_{q}^{0}\right\|_{L^{p_{1}}}+2^{N} 2^{q s}\left\|f_{q}\right\|_{L_{t}^{1} L^{p_{1}}} \\
& +V(t) e^{C V(t)} 2^{2 N} \mu 2^{q(s+2)}\left\|a_{q}\right\|_{L_{t}^{1} L^{p_{1}}} \\
& +2^{2 N} \mu 2^{q s}\left\|\left[\Delta_{q}, v \cdot \nabla\right] a\right\|_{L_{t}^{1} L^{p_{1}}} \\
& +2^{-N} e^{C V(t)} \mu 2^{q(s+2)}\left\|a_{q}\right\|_{L_{t}^{1} L^{p_{1}}} .
\end{aligned}
$$

For low frequencies, $q \leqq N$, we write by Hölder inequality

$$
\mu 2^{q(s+1)}\left\|a_{q}\right\|_{L_{t}^{1} L^{p_{1}}} \leqq(\mu t) 2^{N} 2^{q s}\left\|a_{q}\right\|_{L_{t}^{\infty} L^{p_{1}}} .
$$

Now it remains only the take $\ell^{p_{2}}$ norm. Before that, let us recall that by using Lemma 2 and Minkowski inequality, one can estimate easily

$$
\left\|2^{q s}\right\|\left[\Delta_{q}, v . \nabla\right] a\left\|_{L_{t}^{1} L^{p_{1}}}\right\|_{\ell^{p_{2}}} \precsim\|a\|_{L_{t}^{1} B_{p_{1}, p_{2}}^{s}} \precsim t\|a\|_{{\widetilde{L_{t}^{\infty}}}_{B_{p_{1}, p_{2}}^{s}}} .
$$

Taking then the $\ell^{p_{2}}$ norm, we infer

$$
\begin{aligned}
\mu\|a\|_{\widetilde{L}_{t}^{1} B_{p_{1}, p_{2}}^{s+2}} \precsim & \left\|a^{0}\right\|_{B_{p_{1}, p_{2}}^{s}}+2^{N}\|f\|_{L_{t}^{1} B_{p_{1}, p_{2}}^{s}}+2^{2 N}(V(t)+(\mu t))\|a\|_{{\widetilde{L_{t}^{\infty}}} B_{p_{1}, p_{2}}^{s}} \\
& +\left(V(t) e^{C V(t)} 2^{2 N}+2^{-N} e^{C V(t)}\right) \mu\|a\|_{\widetilde{L}_{t}^{1} B_{p_{1}, p_{2}}^{s+2}} .
\end{aligned}
$$


Choosing $N$ and $t$ such that

$$
V(t) e^{C V(t)} 2^{2 N}+e^{C V(t)} 2^{-N} \precsim \frac{1}{2}
$$

This is possible for small time $t$ such that

$$
V(t) \leqq C_{1},
$$

where $C_{1}$ is a small constant depending on $d$. Under this assumption, one obtains

$$
\mu\|a\| \widetilde{L_{t}^{1} B_{p_{1}, p_{2}}^{s+2}} \precsim\left\|a^{0}\right\|_{B_{p_{1}, p_{2}}^{s}}+C\|f\|_{L_{t}^{1} B_{p_{1}, p_{2}}^{s}}+(1+\mu t)\|a\|_{{\widetilde{L_{t}^{\infty}}}_{B_{p_{1}, p_{2}}^{s}}} .
$$

From the case $r=+\infty$, already shown, we have

$$
\|a\|_{\widetilde{L}_{t}^{\infty} B_{p_{1}, p_{2}}^{s}} \precsim\left\|a^{0}\right\|_{B_{p_{1}, p_{2}}^{s}}+\|f\|_{L_{t}^{1} B_{p_{1}, p_{2}}^{s}}, \quad \text { if } V(t) \leqq C_{1} .
$$

Hence, if $V(t) \leqq C_{1}$ then

$$
\mu\|a\|_{\widetilde{L}_{t}^{1} B_{p_{1}, p_{2}}^{s+2}} \precsim(1+\mu t)\left(\left\|a^{0}\right\|_{B_{p_{1}, p_{2}}^{s}}+\|f\|_{L_{t}^{1} B_{p_{1}, p_{2}}^{s}}\right) .
$$

Since this local result depends only on the size of the Lipschitz norm of the velocity, we can use an iterative argument leading to the required estimate.

\section{Proof of Theorem 1}

This section is devoted to the proof of Theorem 1 which is divided into two parts. In the first one we show, thanks to Theorem 2, the uniform persistence of Besov regularity to Navier-Stokes solution with respect to vanishing viscosity. The second part is dedicated to the proof of the inviscid limit.

\subsection{Uniform persistence of the regularity}

The key for establishing the persistence result is a uniform Lipschitz bound on the velocity. The following proposition gives more, namely

Proposition 2. Let $v^{0} \in B_{p, 1}^{\frac{2}{p}+1}$, with $\left.\left.p \in\right] 2,+\infty\right]$, then Navier-Stokes solution $v_{\mu}$ corresponding to the initial data $v^{0}$ satisfies,

$$
\left\|v_{\mu}(t)\right\|_{B_{\infty, 1}^{1}} \leqq C_{0} e^{C_{0} t}
$$

where $C_{0}$ depends on the initial data but not on the viscosity. 
Proof. Since $B_{p, 1}^{\frac{2}{p}+1} \hookrightarrow B_{\infty, 1}^{1}$ it is sufficient to prove the above estimate for $p=$ $+\infty$. Throughout the proof and for brevity, we will omit the index $\mu$.

By using the dyadic decomposition and Bernstein inequality, one obtains

$$
\|\nabla v(t)\|_{L^{\infty}} \precsim\left\|\Delta_{-1} v(t)\right\|_{L^{\infty}}+\sum_{q \geqq 0}\left\|\Delta_{q} \nabla v(t)\right\|_{L^{\infty}} .
$$

From (1) we see that for high frequencies $q \in \mathbb{N}$,

$$
\left\|\Delta_{q} \nabla v(t)\right\|_{L^{\infty}} \precsim\left\|\Delta_{q} \omega(t)\right\|_{L^{\infty}} .
$$

Hence, it holds that

$$
\|\nabla v(t)\|_{L^{\infty}} \precsim\left\|\Delta_{-1} v(t)\right\|_{L^{\infty}}+\|\omega(t)\|_{B_{\infty, 1}^{0}} .
$$

Now applying Theorem 2, we get

$$
\|\nabla v(t)\|_{L^{\infty}} \precsim\|v(t)\|_{L^{\infty}}+\left\|\omega^{0}\right\|_{B_{\infty, 1}^{0}}\left(1+\int_{0}^{t}\|\nabla v(\tau)\|_{L^{\infty}} \mathrm{d} \tau\right) .
$$

Our next step is to prove that the velocity is bounded for every time. For this purpose we reproduce the idea of [14] used for Euler system. Let $N$ be a positive integer which will be fixed later and define

$$
\Delta_{-N}:=\chi\left(2^{N-1} D\right) \text { and } v_{-N}:=\Delta_{-N} v .
$$

It is obvious that $v_{-N}$ satisfies the equation

$$
\partial_{t} v_{-N}-\mu \Delta v_{-N}=-\Delta_{-N} \mathbb{P}(v \cdot \nabla v),
$$

where $\mathbb{P}$ stands for Leray projector over divergence free vector field.

Hence we obtain in view of parabolic maximum principle and the zero divergence condition

$$
\left\|v_{-N}(t)\right\|_{L^{\infty}} \leqq\left\|v_{-N}(0)\right\|_{L^{\infty}}+\int_{0}^{t}\left\|\Delta_{-N} \mathbb{P} \operatorname{div}(v \otimes v)(\tau)\right\|_{L^{\infty}} \mathrm{d} \tau .
$$

To estimate the integral member, we use the embedding $\dot{B}_{\infty, 1}^{0} \hookrightarrow L^{\infty}$ and the fact that the projector $\mathbb{P}: \dot{B}_{\infty, 1}^{0} \rightarrow \dot{B}_{\infty, 1}^{0}$ is continuous,

$$
\left\|\Delta_{-N} \mathbb{P}(v \cdot \nabla v)(\tau)\right\|_{L^{\infty}} \precsim\left\|\Delta_{-N} \operatorname{div}(v \otimes v)(\tau)\right\|_{\dot{B}_{\infty, 1}^{0}} \precsim 2^{-N}\|v(\tau)\|_{L^{\infty}}^{2} .
$$

From the definition of the vorticity and Bernstein inequality, we obtain

$$
\begin{aligned}
\|v(t)\|_{L^{\infty}} & \leqq\left\|\Delta_{-N} v(t)\right\|_{L^{\infty}}+\left\|\left(\operatorname{Id}-\Delta_{-N}\right) v(t)\right\|_{L^{\infty}} \\
& \leqq\left\|v_{-N}(t)\right\|_{L^{\infty}}+\left\|\left(\operatorname{Id}-\Delta_{-N}\right) \Delta^{-1} \nabla^{\perp} \omega(t)\right\|_{L^{\infty}} \\
& \leqq\left\|v_{-N}(t)\right\|_{L^{\infty}}+C 2^{N}\|\omega(t)\|_{L^{\infty}} \\
& \leqq\left\|v_{-N}(t)\right\|_{L^{\infty}}+C 2^{N}\left\|\omega^{0}\right\|_{L^{\infty}} .
\end{aligned}
$$


In the last estimate we have used the maximum principle:

$$
\|\omega(\tau)\|_{L^{\infty}} \leqq\left\|\omega^{0}\right\|_{L^{\infty}}
$$

Putting together the preceding estimates yields

$$
\|v(t)\|_{L^{\infty}} \precsim\left\|v^{0}\right\|_{L^{\infty}}+\left\|\omega^{0}\right\|_{L^{\infty}} 2^{N}++2^{-N} \int_{0}^{t}\|v(\tau)\|_{L^{\infty}}^{2} \mathrm{~d} \tau .
$$

By choosing $N$ such that

$$
2^{2 N} \simeq 1+\frac{\int_{0}^{t}\|v(\tau)\|_{L^{\infty}}^{2} \mathrm{~d} \tau}{\left\|\omega^{0}\right\|_{L^{\infty}}}
$$

we get

$$
\|v(t)\|_{L^{\infty}}^{2} \leqq C\left\|v^{0}\right\|_{L^{\infty}}^{2}+C\left\|\omega^{0}\right\|_{L^{\infty}}^{2}+\left\|\omega^{0}\right\|_{L^{\infty}} \int_{0}^{t}\|v(\tau)\|_{L^{\infty}}^{2} \mathrm{~d} \tau .
$$

According to Gronwall's inequality this gives

$$
\|v(t)\|_{L^{\infty}} \leqq C\left(\left\|v^{0}\right\|_{L^{\infty}}+\left\|\omega^{0}\right\|_{L^{\infty}}\right) e^{C\left\|\omega^{0}\right\|_{L^{\infty}}} .
$$

Coming back to (19) we obtain

$$
\|\nabla v(t)\|_{L^{\infty}} \leqq C_{0} e^{C_{0} t}+C\left\|\omega^{0}\right\|_{B_{\infty, 1}^{0}}\left(1+\int_{0}^{t}\|\nabla v(\tau)\|_{L^{\infty}} \mathrm{d} \tau\right),
$$

and Gronwall's inequality yields the required estimate.

Let us now achieve the proof of the persistence of Besov regularity.

Applied to the viscous vorticity $\omega_{\mu}$ Theorem 3 gives

$$
\begin{aligned}
\left\|\omega_{\mu}(t)\right\|_{B_{p, 1}^{\frac{2}{p}}} \leqq\left\|\omega_{\mu}\right\|_{\widetilde{L}_{t}^{\infty}} B_{p, 1}^{\frac{2}{p}} & \leqq C\left\|\omega^{0}\right\|_{B_{p, 1}^{\frac{2}{p}}} e^{C \int_{0}^{t}\left\|\nabla v_{\mu}(\tau)\right\|_{L^{\infty}} \mathrm{d} \tau} \\
& \leqq C_{0} e^{\exp C_{0} t}
\end{aligned}
$$

In the last line we have used the Lipschitz bound (18). Finally, to estimate the velocity, we write

$$
\begin{aligned}
\left\|v_{\mu}(t)\right\|_{B_{p, 1}^{\frac{2}{p}+1}} & \leqq\left\|\Delta_{-1} v_{\mu}(t)\right\|_{L^{p}}+C\left\|\omega_{\mu}(t)\right\|_{B_{p, 1}^{\frac{2}{p}}} \\
& \leqq C\left\|v_{\mu}(t)\right\|_{L^{p}}+C_{0} e^{\exp C_{0} t} .
\end{aligned}
$$

We are reduced to estimate the $L^{p}$ norm of the viscous velocity. We have to consider only the case of $p \in] 2,+\infty[$ (remember (20) for $p=+\infty)$. The $L^{p}$ energy estimate of Navier-Stokes system

$$
\left\|v_{\mu}(t)\right\|_{L^{p}} \leqq\left\|v_{\mu}^{0}\right\|_{L^{p}}+\int_{0}^{t}\left\|\mathbb{P}\left(v_{\mu} \cdot \nabla v_{\mu}\right)(\tau)\right\|_{L^{p}} \mathrm{~d} \tau .
$$


From the boundedness of Riesz transform, we infer

$$
\left\|\mathbb{P}\left(v_{\mu} \cdot \nabla v_{\mu}\right)(t)\right\|_{L^{p}} \leqq\left\|\mathbb{P}\left(v_{\mu} \cdot \nabla v_{\mu}\right)(t)\right\|_{\dot{B}_{p, 1}^{0}} \precsim\left\|v_{\mu} \cdot \nabla v_{\mu}(t)\right\|_{\dot{B}_{p, 1}^{0}} \cdot
$$

In view of Lemma 4 of the appendix and (18)

$$
\left\|v_{\mu} \cdot \nabla v_{\mu}(t)\right\|_{\dot{B}_{p, 1}^{0}} \precsim\left\|v_{\mu}(t)\right\|_{L^{p}}\left\|v_{\mu}(t)\right\|_{B_{\infty, 1}^{1}} \leqq C_{0} e^{C_{0} t}\left\|v_{\mu}(t)\right\|_{L^{p}} .
$$

Combining both last estimates and using Gronwall's inequality we get

$$
\left\|v_{\mu}(t)\right\|_{L^{p}} \leqq C_{0} e^{\exp C_{0} t}
$$

Therefore, we obtain for all $t \in \mathbb{R}_{+}$,

$$
\left\|v_{\mu}(t)\right\|_{B_{p, 1}^{\frac{2}{p}+1}} \leqq C_{0} e^{\exp C_{0} t} .
$$

It is easy to check that the same proof works for the Eulerian velocity, and we have

$$
\|v(t)\|_{B_{\infty, 1}^{1}} \leqq C_{0} e^{C_{0} t}
$$

\subsection{Convergence rate}

In this subsection we give the proof of the inviscid limit. The following proposition gives more than Theorem 1 , namely we can replace Lebesgue space $L^{p}$ by the subspace $B_{p, 1}^{0}$.

Proposition 3. Let $p \in] 2,+\infty]$ and $v^{0}$ be a divergence free vector field belonging to $B_{p, 1}^{\frac{2}{p}+1}$. Then Navier-Stokes solution and Euler one satisfy, globally in time,

$$
\left\|\left(v_{\mu}-v\right)(t)\right\|_{B_{p, 1}^{0}} \leqq C_{0} e^{\exp C_{0} t}(\mu t)^{\frac{1}{2}+\frac{1}{p}}(1+\mu t)^{\frac{1}{2}-\frac{1}{p}} .
$$

Proof. We set

$$
w_{\mu}=v_{\mu}-v \text { and } \bar{\pi}_{\mu}=\pi_{\mu}-\pi .
$$

Obviously $\left(w_{\mu}, \bar{\pi}_{\mu}\right)$ is solution of

$$
\left(\widetilde{N S}_{\mu}\right) \quad\left\{\begin{array}{l}
\partial_{t} w_{\mu}+v_{\mu} \cdot \nabla w_{\mu}=\mu \Delta v_{\mu}-w_{\mu} \cdot \nabla v-\nabla \bar{\pi}_{\mu} \\
w_{\mu}(0)=0
\end{array}\right.
$$

According to Theorem 2, we have

$$
\left\|w_{\mu}(t)\right\|_{B_{p, 1}^{0}} \precsim\left(1+V_{\mu}(t)\right) \int_{0}^{t}\left(\mu\left\|\Delta v_{\mu}\right\|_{B_{p, 1}^{0}}+\left\|w_{\mu} \cdot \nabla v\right\|_{B_{p, 1}^{0}}+\left\|\nabla \bar{\pi}_{\mu}\right\|_{B_{p, 1}^{0}}\right) \mathrm{d} \tau .
$$

To estimate the first integral term of the right-hand side, we write from (1) and (3)

$$
\left\|\Delta v_{\mu}\right\|_{L_{t}^{1} B_{p, 1}^{0}} \leqq C\left\|\omega_{\mu}\right\|_{\widetilde{L}_{t}^{1} B_{p, 1}^{1}} \leqq C\left\|\omega_{\mu}\right\|_{\widetilde{L_{t}^{1}} B_{p, \infty}^{\frac{2}{p}}}^{\frac{1}{2}+\frac{1}{p}}\left\|\omega_{\mu}\right\|_{\widetilde{L}_{t}^{1} B_{p, \infty}^{2+\frac{2}{p}}}^{\frac{1}{2}-\frac{1}{p}} .
$$


Applying Hölder inequality and estimate (21) yields

$$
\begin{aligned}
\left\|\omega_{\mu}\right\|_{\widetilde{L_{t}^{1}} B_{p, \infty}^{\frac{2}{p}}}^{\frac{1}{2}+\frac{1}{p}} & \leqq t^{\frac{1}{2}+\frac{1}{p}}\left\|\omega_{\mu}\right\|_{\widetilde{L_{t}^{\infty}} B_{p, 1}^{\frac{1}{2}}}^{\frac{1}{p}} \\
& \leqq C_{0} t^{\frac{1}{2}+\frac{1}{p}} e^{\exp C_{0} t} .
\end{aligned}
$$

On the other hand Theorem 3 gives

$$
\begin{aligned}
&\left\|\omega_{\mu}\right\|_{\widetilde{L_{t}^{1}} B_{p, \infty}^{2+\frac{2}{p}}} \leqq \mu^{-1} e^{C V_{\mu}(t)}(1+\mu t)\left\|\omega^{0}\right\|_{B_{p, 1}^{\frac{2}{p}}} \\
& \leqq C_{0} e^{\exp C_{0} t} \mu^{-1}(1+\mu t) .
\end{aligned}
$$

Thus, we infer

$$
\mu\left\|\Delta v_{\mu}\right\|_{L_{t}^{1} B_{p, 1}^{0}} \leqq C_{0} e^{\exp C_{0} t}(\mu t)^{\frac{1}{2}+\frac{1}{p}}(1+\mu t)^{\frac{1}{2}-\frac{1}{p}} .
$$

To bound the second term of the right-hand side of (23), we use Lemma 4.

$$
\left\|w_{\mu} \cdot \nabla v(t)\right\|_{B_{p, 1}^{0}} \leqq C\left\|w_{\mu}(t)\right\|_{B_{p, 1}^{0}}\|v(t)\|_{B_{\infty, 1}^{1}} .
$$

So the inequality (22) yields

$$
\left\|w_{\mu} \cdot \nabla v(t)\right\|_{B_{p, 1}^{0}} \leqq C_{0} e^{C_{0} t}\left\|w_{\mu}(t)\right\|_{B_{p, 1}^{0}} .
$$

For the pressure we use the following identity based on $\operatorname{div} v=\operatorname{div} w_{\mu}=0$

$$
\operatorname{div}\left(v \cdot \nabla w_{\mu}\right)=\operatorname{div}\left(w_{\mu} \cdot \nabla v\right) .
$$

Applying the divergence operator to the system $\widetilde{\left(N S_{\mu}\right)}$, we find that

$$
\Delta \bar{\pi}_{\mu}=\operatorname{div}\left(w_{\mu} \cdot \nabla v_{\mu}+v \cdot \nabla w_{\mu}\right) .
$$

Thus, we infer

$$
-\Delta \bar{\pi}_{\mu}=\operatorname{div}\left(w_{\mu} \cdot \nabla\left(v_{\mu}+v\right)\right) .
$$

This yields in view of the embedding $\dot{B}_{p, 1}^{0} \hookrightarrow B_{p, 1}^{0}$ and the boundedness of Riesz transform

$$
\left\|\nabla \bar{\pi}_{\mu}\right\|_{B_{p, 1}^{0}} \leqq C\left\|w_{\mu} \cdot \nabla\left(v_{\mu}+v\right)\right\|_{\dot{B}_{p, 1}^{0}} .
$$

Now we use successively Lemma 4 and estimates (18) and (22)

$$
\begin{aligned}
\left\|\nabla \bar{\pi}_{\mu}\right\|_{B_{p, 1}^{0}} & \leqq C\left\|w_{\mu}\right\|_{B_{p, 1}^{0}}\left\|v_{\mu}+v\right\|_{B_{\infty, 1}^{1}} \\
& \leqq C_{0} e^{C_{0} t}\left\|w_{\mu}\right\|_{B_{p, 1}^{0}} .
\end{aligned}
$$

Plugging (26), (25) and (24) into (23) yields

$$
\left\|w_{\mu}(t)\right\|_{B_{p, 1}^{0}} \leqq C_{0} e^{\exp C_{0} t}(\mu t)^{\frac{1}{2}+\frac{1}{p}}(1+\mu t)^{\frac{1}{2}-\frac{1}{p}}+C_{0} e^{C_{0} t} \int_{0}^{t}\left\|w_{\mu}(\tau)\right\|_{B_{p, 1}^{0}} \mathrm{~d} \tau .
$$

To complete the proof we use Gronwall's inequality. 


\section{Appendix}

The aim of this section is to prove the following result.

Lemma 4. Let $v$ be a divergence free vector field belonging to $B_{p, 1}^{0}$ and $f$ a function of the space $B_{\infty, 1}^{1}$. Then the following estimate occurs

$$
\|v \cdot \nabla f\|_{B_{p, 1}^{0}} \leqq C\|v\|_{B_{p, 1}^{0}}\|f\|_{B_{\infty, 1}^{1}} .
$$

We have, in addition, the following precise result

$$
\|v \cdot \nabla f\|_{\dot{B}_{p, 1}^{0}} \leqq C\|v\|_{B_{p, 1}^{0}}\|f\|_{B_{\infty, 1}^{1}} .
$$

Besides if $f=v$ then we have

$$
\|v \cdot \nabla v\|_{\dot{B}_{p, 1}^{0}} \leqq C\|v\|_{L^{p}}\|v\|_{B_{\infty, 1}^{1}} .
$$

Proof. We shall now give the proof of Lemma 4 which is based on Bony's decomposition [1]. We write

$$
v \cdot \nabla f=T_{v} \cdot \nabla f+T_{\nabla f} \cdot v+R(v, \nabla f) .
$$

By definition of the paraproduct, we have

$$
\begin{aligned}
\left\|T_{v} \cdot \nabla f\right\|_{B_{p, 1}^{0}} & \leqq C \sum_{q \in \mathbb{N}}\left\|S_{q-1} v\right\|_{L^{p}}\left\|\Delta_{q} \nabla f\right\|_{L^{\infty}} \\
& \leqq C\|v\|_{B_{p, 1}^{0}} \sum_{q \in \mathbb{N}} 2^{q}\left\|\Delta_{q} f\right\|_{L^{\infty}} \\
& \leqq C\|v\|_{B_{p, 1}^{0}}\|f\|_{B_{\infty, 1}^{1}} .
\end{aligned}
$$

By the same way, we show that

$$
\left\|T_{\nabla f} \cdot v\right\|_{B_{p, 1}^{0}} \leqq C\|v\|_{B_{p, 1}^{0}}\|f\|_{B_{\infty, 1}^{1}} .
$$

To estimate the last term, we use the incompressibility hypothesis

$$
R(v, \nabla f)=\operatorname{div} R(v, f) .
$$

So we have by definition of the remainder $R$

$$
\|\operatorname{div} R(v, f)\|_{B_{p, 1}^{0} \leqq} \sum_{q \geqq-1}\left\|\operatorname{div} \Delta_{q} \sum_{\substack{j \geqq-1 \\ i \in\{\overline{0}, \mp 1\}}} \Delta_{j} v \Delta_{j+i} f\right\|_{L^{p}} .
$$

Using the fact that the Fourier transform of $\Delta_{j} v \Delta_{j+i} f$ is supported in a ball of radius $2^{j}$, so we get, in view of Bernstein lemma

$$
\begin{aligned}
\|\operatorname{div} R(v, f)\|_{B_{p, 1}^{0}} & \leqq C \sum_{i \in\{0 ; \mp 1\}} \sum_{q \geqq-1} 2^{q} \sum_{j \geqq q-M}\left\|\Delta_{j} v\right\|_{L^{p}}\left\|\Delta_{j+i} f\right\|_{L^{\infty}} \\
& \leqq C\|f\|_{B_{\infty, 1}^{1}} \sum_{q \geqq-1} 2^{q} \sum_{j \geqq q-M} 2^{-j}\left\|\Delta_{j} v\right\|_{L^{p}} .
\end{aligned}
$$


Since $\ell^{1}$ is an algebra space under the convolution law then the wanted result is proved.

Let us now prove the second estimate. We separate low an high frequencies. For low frequencies we use Bernstein inequality; however for high frequencies we make appeal to the first result of Lemma 4:

$$
\begin{aligned}
\|v \cdot \nabla f\|_{\dot{B}_{p, 1}^{0}} & \leqq \sum_{j \leqq-1}\left\|\dot{\Delta}_{j}(v \cdot \nabla f)\right\|_{L^{p}}+C\|v \cdot \nabla f\|_{B_{p, 1}^{0}} \\
& \leqq C\|v\|_{L^{p}}\|f\|_{L^{\infty}}+C\|v\|_{B_{p, 1}^{0}}\|f\|_{B_{\infty, 1}^{1}} \\
& \leqq C\|v\|_{B_{p, 1}^{0}\|f\|_{B_{\infty, 1}^{1}} .}
\end{aligned}
$$

The reader can verify that the preceding arguments can be adapted without difficulties to show the last estimate of Lemma 4.

\section{References}

1. Bony, J.-M.: Calcul symbolique et propagation des singularités pour les équations aux dérivées partielles non linéaires. Ann. Ecole Norm. Sup. 14, 209-246 (1981)

2. Beale, J.T., Kato, T., Majda, A.: Remarks on the breakdown of smooth solutions for 3-D Euler equations. Commun. Math. Phys. 94, 61-66 (1984)

3. Chat, D.: Local existence and blow-up criterion for the Euler equations in the Besov spaces. Asymptot. Anal. 38(3-4), 339-358 (2004)

4. Chemin, J.-Y.: Perfect incompressible Fluids. Oxford University Press, New York, 1998

5. Chemin, J.-Y.: Théorèmes d'unicité pour le système de Navier-Stokes tridimensionnel. J. Anal. Math. 77, 27-50 (1999)

6. Danchin, R.: Poches de tourbillon visqueuses. J. Math. Pures Appl. (9) 76(7), 609-647 (1997)

7. Fujita, H., Kato, T.: On the nonstationnary Navier-Stokes system. Rend. Sem. Mat. Univ. Padova, 32, 243-260 (1962)

8. Hмidi, T.: Régularité höldérienne des poches de tourbillon visqueuses. J. Math. Pures Appl. (9) 84(11), 1455-1495 (2005)

9. Hmidi, T., Keraani, S.: Inviscid limit for the two-dimensional Navier-Stokes system in a critical Besov space. Asymptotic Anal. 53(3), 125-138 (2007)

10. Hmidi, T., Keraani, S.: Existence globale pour le système d'Euler incompressible 2-D dans $B_{\infty, 1}^{1}$. C. R. Math. Acad. Sci. Paris 341(11), 655-658 (2005)

11. Kato, T., Ponce, G.: Commutator estimates and the Euler and Navier-Stokes equations. Commun. Pure Appl. Math. 41, 891-907 (1988)

12. Leray, J.: Sur le mouvement d'un liquide visqueux remplissant l'espace. Acta Math. 63, 193-248 (1934)

13. MajdA, A.: Vorticity and the mathematical theory of an incompressible fluid flow. Commun. Pure Appl. Math. 38, 187-220 (1986)

14. Serfati, P.: Solutions $C^{\infty}$ en temps, $n$-log Lipschitz bornées en espace et équation d'Euler. C. R. Acad. Sci. Paris 320(5), 555-558 (1995)

15. Vishik, M.: Hydrodynamics in Besov Spaces. Arch. Rational Mech. Anal 145, 197-214 (1998) 